\section{Autologous stem cell transplantation is safe and effective for fit, older myeloma patients: exploratory results from the Myeloma XI trial}

\author{
Charlotte Pawlyn, ${ }^{1,2}$ David A. Cairns, ${ }^{3}$ Tom Menzies, ${ }^{3}$ John R. Jones, ${ }^{4}$ Matthew \\ W. Jenner, ${ }^{5}$ Gordon Cook, ${ }^{3,6}$ Kevin D. Boyd, ${ }^{2}$ Mark T. Drayson, ${ }^{7}$ Martin F. \\ Kaiser, ${ }^{1,2}$ Roger G. Owen, ${ }^{8}$ Walter Gregory, ${ }^{3}$ Gareth J. Morgan, ${ }^{9}$ Graham H. \\ Jackson $^{10}$ and Faith E. Davies ${ }^{9}$ on behalf of the UK NCRI Haemato-Oncology \\ Clinical Studies Group.
}

${ }^{1}$ The Institute of Cancer Research, London, UK; ${ }^{2}$ The Royal Marsden Hospital, London, UK; ${ }^{3}$ Clinical Trials Research Unit, Leeds Institute of Clinical Trials Research, University of Leeds, Leeds, UK; ' ${ }^{4}$ King's Hospital NHS Foundation Trust, London, UK; ${ }^{5}$ University Hospital Southampton NHS Foundation Trust, Southampton, UK; ' ${ }^{\circ}$ eeds Cancer Centre, Leeds Teaching Hospitals Trust, Leeds, UK; ${ }^{7}$ Institute of Immunology and Immunotherapy, University of Birmingham, Birmingham, UK; ${ }^{8} \mathrm{HMDS}$, Leeds Cancer Centre, Leeds Teaching Hospitals Trust, Leeds, UK; ${ }^{P}$ Perlmutter Cancer Center, NYU Langone, New York, NY, USA and ${ }^{10}$ Department of Haematology, Newcastle University, Newcastle, UK

\section{ABSTRACT}

A utologous stem cell transplant (ASCT) remains the standard of care for consolidation after induction therapy for eligible patients with newly diagnosed myeloma. In recent clinical trials comparing ASCT to delayed ASCT, patients aged over 65 were excluded. In real-world practice stem cell transplants are not restricted to those aged under 65 and clinicians decide on transplant eligibility based on a patient's fitness rather than a strict age cut-off. Data from the UK NCRI Myeloma XI trial, a large phase III randomized controlled trial with pathways for transplant-eligible and -ineligible patients, were used in an exploratory analysis to examine the efficacy and toxicity of ASCT in older patients including an analysis using an age-matched population to compare outcomes for patients receiving similar induction therapy with or without ASCT. Older patients within the transplant-eligible pathway were less likely to undergo stem cell harvest at the end of induction than younger patients and of those patients undergoing ASCT there was a reduction in progression-free survival associated with increasing age. ASCT in older patients was well tolerated with no difference in morbidity or mortality between patients aged $<65,65-69$ and $70-75$ years. In an age-matched population of patients including those in both the transplant-eligible and -ineligible pathways there was a significant advantage associated with undergoing ASCT with increases in progression-free survival (hazard ratio $0.41, P<0.0001$ ) and overall survival (hazard ratio $0.51, P<0.0001)$, which persisted even after adjustment for baseline covariates including those related to frailty and response to induction. These findings support the use of ASCT for selected fit, older myeloma patients. EudraCT number, 2009-010956-93

\section{Introduction}

Autologous stem cell transplant (ASCT) is delivered as consolidation after induction therapy for eligible patients with newly diagnosed myeloma. The use of ASCT became standard of care based on several randomized controlled trials that demonstrated progression-free (PFS) and overall survival (OS) benefits. ${ }^{1.4}$ The ongoing use of ASCT in the context of current induction treatment regimens continues to be supported by data from two recent large phase III studies. ${ }^{5,6}$ Both these studies, however, excluded patients aged over 65 years old. In real-world practice stem cell transplants are not restricted to those aged under 65 and clinicians decide on transplant eligibility

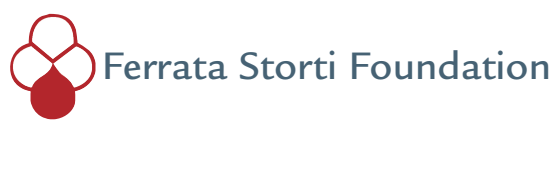

Haematologica 2022

Volume 107(1):231-242

Correspondence:

CHARLOTTE PAWLYN

charlotte.pawlyn@icr.ac.uk

Received: June 7, 2020.

Accepted: November 25, 2020.

Pre-published: December 3, 2020.

https://doi.org/10.3324/haematol.2020.262360

(C)2022 Ferrata Storti Foundation

Material published in Haematologica is covered by copyright. All rights are reserved to the Ferrata Storti Foundation. Use of published material is allowed under the following terms and conditions:

https://creativecommons.org/licenses/by-nc/4.0/legalcode. Copies of published material are allowed for personal or internal use. Sharing published material for non-commercial purposes is subject to the following conditions:

https://creativecommons.org/licenses/by-nc/4.0/legalcode, sect. 3. Reproducing and sharing published material for commercial purposes is not allowed without permission in writing from the publisher. 
based on the individual patient's fitness rather than a strict age cut-off. Standard-of-care conditioning for ASCT consists of melphalan given at a dose of $200 \mathrm{mg} / \mathrm{m}^{2}$ although a lower dose of $140 \mathrm{mg} / \mathrm{m}^{2}$ may be delivered in the case of renal impairment and is sometimes considered by clinicians for the treatment of older patients.

The European Bone Marrow Transplant (EBMT) registry has documented an increase in the number of patients aged over 65 undergoing ASCT in recent years. Between 20012005, 2,478 patients aged 65-69 years underwent ASCT, constituting $14.1 \%$ of transplanted patients, and the number rose to 3,860 (15.8\%) in the years 2006-2010. A similar pattern was seen for those aged 70 or over: $497(2.8 \%)$ in 2001-2005 and 740 (3\%) in 2006-2010.' In this analysis there was no apparent difference in transplant-related mortality between those aged 60-64, 65-69 and $\geq 70$ years, with the rates being $1.8 \%, 2.1 \%$ and $2.4 \%$, respectively. This trend is mirrored in data from the USA-based Center for International Blood and Marrow Transplant Research (CIBMTR). ${ }^{8}$

Two randomized studies of ASCT in older patients were conducted using dose-reduced melphalan $\left(100 \mathrm{mg} / \mathrm{m}^{2}\right)$ tandem transplantation following conventional chemotherapy induction regimens. The first study randomized patients aged 50-70 years between melphalan prednisolone (MP) and vincristine, doxorubicin and dexamethasone for two cycles followed by dose-reduced melphalan and ASCT for two cycles (VAD+ASCT100). ${ }^{9}$ The use of ASCT was associated with improved event-free survival and OS. The second study randomized patients between MP, MP plus thalidomide and VAD+ASCT100. This study demonstrated an improvement in OS for VAD+ASCT100 versus MP but the use of MP plus thalidomide was superior to both approaches. ${ }^{10}$ These data supported the use of ASCT100 in the context of conventional chemotherapy induction in older patients but the combination of both immunomodulatory agent induction and ASCT was not examined.

Several previous retrospective studies have examined outcomes following ASCT for patients over the age of 65 or 70 following immunomodulatory and/or proteasome inhibitor-based induction. A large retrospective study of patients treated at the Mayo Clinic (USA) compared 207 patients aged 70 and over to 1,765 patients aged less than $70 .{ }^{11}$ There was no significant difference in PFS, OS or transplant-related mortality between the groups. A similar analysis of patients treated in Heidelberg (Germany), found no difference between outcomes for those aged 60-64, 6569 or 70-75 years. ${ }^{12}$ Retrospective data analysis has also been used to compare ASCT and no-ASCT treatment strategies in small cohorts of patients over the age of $65.13,14$ These studies support the use of ASCT in patients over the age of 65 thought to be fit, but did not address whether ASCT is preferred over conventional therapy for patients in this older age group. To our knowledge, no randomized comparison of ASCT to no-ASCT has been undertaken in patients over the age of 65 in the current treatment landscape.

Data from the UK National Cancer Research Institute (NCRI) Myeloma XI trial, a large phase III randomized controlled trial with pathways for transplant-eligible (TE) and ineligible (TNE) patients, was used to explore the efficacy and toxicity of ASCT in older patients including an analysis using an age-matched population. ${ }^{15,16}$ Patients in the trial were randomized between induction treatment with thalidomide- or lenalidomide-based triplets, with the same combinations being used in both the TE and TNE pathways. This gives the opportunity to examine outcomes for TE patients of different ages, but also to compare outcomes for similar patients receiving the same induction therapy with or without ASCT.

\section{Methods}

Myeloma XI is a phase III, open-label, parallel-group, multi-arm, adaptive trial and recruited newly diagnosed patients of all ages. Eligible patients were aged $\geq 18$ years. The trial was designed to reflect a population as close to real-world as was considered safe. Exclusion criteria were therefore limited, but included previous treatment for myeloma (excluding local radiotherapy, bisphosphonates, and corticosteroids), previous or concurrent malignancies (including myelodysplastic syndromes), grade $\geq 2$ peripheral neuropathy, acute renal failure (unresponsive to up to $72 \mathrm{~h}$ of rehydration, characterized by creatinine $>500 \mu \mathrm{mol} / \mathrm{L}$ or urine output $<400 \mathrm{~mL} /$ day or requiring dialysis), and active or prior hepatitis C virus infection. There were separate pathways for TE and TNE patients.

The trial was performed in accordance with the Declaration of Helsinki 1996, and the study was approved by the national ethics review board (National Research Ethics Service, London, UK), institutional review boards of the participating centers, and the competent regulatory authority (Medicines and Healthcare Products Regulatory Agency, London, UK). All patients provided written informed consent. The trial was registered with the EU Clinical Trials Register (EudraCT number, 2009-010956-93).

The details of the trial therapy and most primary outcomes have been published already. ${ }^{15,16}$ In brief, patients in both pathways were randomized between a thalidomide-containing triplet (cyclophosphamide, thalidomide and dexamethasone) or a lenalidomide-containing triplet (cyclophosphamide, lenalidomide and dexamethasone). Induction treatment was given for a minimum of four cycles (in the TE pathway) or six cycles (TNE) and to maximum response, and there was an induction intensification question for those with a suboptimal response to initial induction. All TE patients were planned to undergo an ASCT. Patients in both pathways underwent maintenance randomization between lenalidomide ( \pm vorinostat) and observation.

The choice of pathway, TE or TNE, was left to the local investigator based on co-morbidities and the patient's/clinician's preference. There was no age limit for entry into the TE pathway. Induction therapy in the TNE pathway was administered with an attenuation of dexamethasone dosing (Online Supplementary Table S1) but was otherwise similar between pathways.

All participants in the intensive pathway who responded (with at least a minimal response) to induction chemotherapy were planned to go on to receive high-dose melphalan and ASCT. Peripheral blood stem cell harvest was planned to commence after the participant had completed the induction and intensification (if applicable) treatment. Stem cell mobilization and stem cell harvest were performed according to local practice but with advice to aim for the collection of enough stem cells for at least two transplants. High-dose melphalan and ASCT were given according to local practice. Adjustment for renal insufficiency was advised. Participants with serum creatinine $<200 \mu \mathrm{mol} / \mathrm{L}$ prior to transplantation were to receive the standard dose of $200 \mathrm{mg} / \mathrm{m}^{2}$ melphalan while those with serum creatinine $>200 \mu \mathrm{mol} / \mathrm{L}$ were to receive $140 \mathrm{mg} / \mathrm{m}^{2}$. There was no recommendation to reduce the melphalan dose based on age in the protocol.

This is a retrospective, exploratory analysis of data from the Myeloma XI trial. For the first set of analyses patients in the TE 
pathway were categorized by age group $<65,65-69$ and 70-75 and their baseline characteristics, treatment and harvest data summarized. PFS and OS, measured from baseline trial randomization, were compared between age groups using the Kaplan-Meier method. Comparisons were made between the allocated groups using the Cox proportional hazards model stratified by the minimization stratification factors, excluding center, and hazard ratios (HR) and 95\% confidence intervals (95\% CI) were estimated. The frequencies of reported serious adverse events and patients' deaths were examined to compare transplant-related morbidity and mortality between age groups.

Relative survival estimates were obtained using flexible parametric survival models on the hazard scale with four degrees of freedom ${ }^{17}$ with the same covariates included in the model. Relative survival was defined as the observed survival divided by the expected survival where the expected survival was obtained from national life tables stratified by age at diagnosis, sex and calendar year. UK life-time risk was estimated from data available from the Office for National Statistics. ${ }^{18}$

In order to compare outcomes between patients undergoing ASCT or not, a second set of analyses was performed using an age-matched group of patients in the TE and TNE pathways. This was defined by overlap of the age distributions in each pathway and comprised the older patients within the TE pathway and younger patients within the TNE pathway but excluded the extremes in both pathways. The optimal overlap was of patients aged 64-70 within each pathway, which was chosen with the aim of maximizing the number of patients in the analysis while achieving a balance between the patients receiving ASCT (52.5\%) and those who did not receive ASCT (47.5\%). Three groups were considered: (i) TE patients who underwent ASCT (TE-ASCT); (ii) TE patients who did not undergo ASCT (TE-noASCT) and (iii) TNE patients (who did not undergo ASCT). The characteristics of the patients in the three groups at entry into the trial were summarized. Patients were scored according to the UK Myeloma Research Alliance Myeloma Risk Profile (MRP) ${ }^{19}$ and the proportion of patients in each of the groups compared. When analyzing time-to-event outcomes in order to avoid a survivor or immortal time bias that would be incurred by comparing all patients within these groups from baseline, patients were only included if they remained eligible to continue in the trial at the end of induction \pm intensification and their outcomes were measured from the end of induction \pm intensification therapy. This time-point was close to the ASCT date for the patients in the TE-ASCT group, but represents a common time-point that could be identified in all patients to enable comparison.

To estimate the treatment effect of ASCT as compared to no ASCT in this subgroup, propensity score weighting using inverse probability of treatment weights was implemented. Propensity score weighting is a useful tool to account for imbalances in observed confounders between groups when estimating treatment effects from non-randomized data. A propensity score is a single score that represents the probability of receiving a treatment, conditional on a set of observed covariates. The goal of creating a propensity score is to balance covariates between individuals who did and did not receive a treatment, making it easier to isolate the effect of a treatment. The propensity score was based on a patient's age, sex, World Health Organization (WHO) performance status, International Staging System (ISS) stage, induction treatment and response after completing induction treatments. The propensity score was applied using normalized inverse probability of treatment weighting (IPTW). In IPTW, each treated subject (ASCT) receives a weight equal to the inverse of the propensity score, and each control subject (no ASCT) receives a weight equal to the inverse of one minus the propensity score.
For the IPTW analysis, probability weights were applied to the individual participants' data for calculation of the survivor function estimate and partial likelihood in the Cox model. Statistical analysis was performed using SAS v9.4 (SAS Institute Inc., Cary, NC, USA) and Stata IC v16 (StataCorp. College Station, TX, USA).

PFS was defined as the time from the point stated above to the date of confirmed disease progression or death from any cause. OS was defined as the time from the point stated above to the date of death from any cause. Cytogenetic profiling was performed using multiplex ligation-dependent probe amplification and quantitative real-time polymerase chain reaction analysis..$^{20,21}$ Cytogenetic risk was defined as standard when there were no adverse lesions, high in the presence of $t(4 ; 14), t(14 ; 16), t(14 ; 20)$, $\operatorname{del}(17 p)$, or gain $(1 q)$, and ultra-high when more than one adverse lesion was present. ${ }^{22,23}$ The data cut-off for inclusion in this analysis was May 31, 2019.

\section{Results}

Outcomes for transplant-eligible pathway patients by age

The 2,042 patients enrolled in the TE pathway had a median age of 61 (28-75) years; 546 (27\%) were aged 65-69 and $101(5 \%)$ were aged 70-75 (Table 1). Older patients were more frequently categorized as ISS stage III and had a lower performance status than younger patients. There was no significant difference in the proportion of patients in each of the cytogenetic risk groups or the number of patients in each arm of the induction treatment randomization between age groups. Response at the end of induction was similar across age groups.

Older patients in the TE pathway were less likely than younger patients to undergo stem cell harvest at the end of induction. The percentage of patients undergoing stem cell harvest fell from $73.5 \%$ in those aged $<65$ years, to $62.2 \%$ among those aged $65-69$ and only $57.4 \%$ in the group aged 70 or older (Table 1, Online Supplementary Figure S1). The reason given for not proceeding to stem cell harvest and subsequent transplantation was more likely to be due to the clinician/patient not considering that they were fit enough to proceed in the older age groups than in the younger patients (Table 1).

Older patients had a lower median harvested $\mathrm{CD} 34^{+}$cell count but still had a high rate of achieving the standard cutoff of $2 \times 10^{6} \mathrm{CD}_{3} 4^{+}$cells $/ \mathrm{kg}$ needed for one ASCT. The percentage of patients achieving this target was $95.0 \%$ in those aged $<65,90.0 \%$ in those aged $65-69$ and $88.7 \%$ among patients aged 70 or older. Conversely, fewer patients in the older age groups achieved the cut-off of $4 \times 10^{6} \mathrm{CD}^{4} 4^{+}$ cells $/ \mathrm{kg}$ considered adequate for two ASCT, with reductions from $63.4 \%$ to $45.6 \%$ to $32.1 \%$ in the respective age groups.

Of the 2,042 patients in the TE pathway, 1,370 (67\%) received melphalan. Most patients $(84.7 \%)$ received 200 $\mathrm{mg} / \mathrm{m}^{2}$ with only $10.5 \%$ reported to have received 140 $\mathrm{mg} / \mathrm{m}^{2}$. The proportion receiving the lower dose increased in the older age groups with the $140 \mathrm{mg} / \mathrm{m}^{2}$ dose being given to only $5.5 \%$ of patients aged $<65$ years, but $19.9 \%$ of those aged $65-69$ and $45.5 \%$ of those aged $>70$ years. This appeared to be due to both an increased incidence of elevated serum creatinine $(>200 \mu \mathrm{mol} / \mathrm{L})$ in the older age groups and the systematic use of the lower dose for older patients in some centers.

Response to transplant, PFS and OS outcomes for patients of different ages within the TE pathway who underwent 
Table 1. Baseline characteristics and treatment received for patients within the transplant-eligible pathway and by age group.

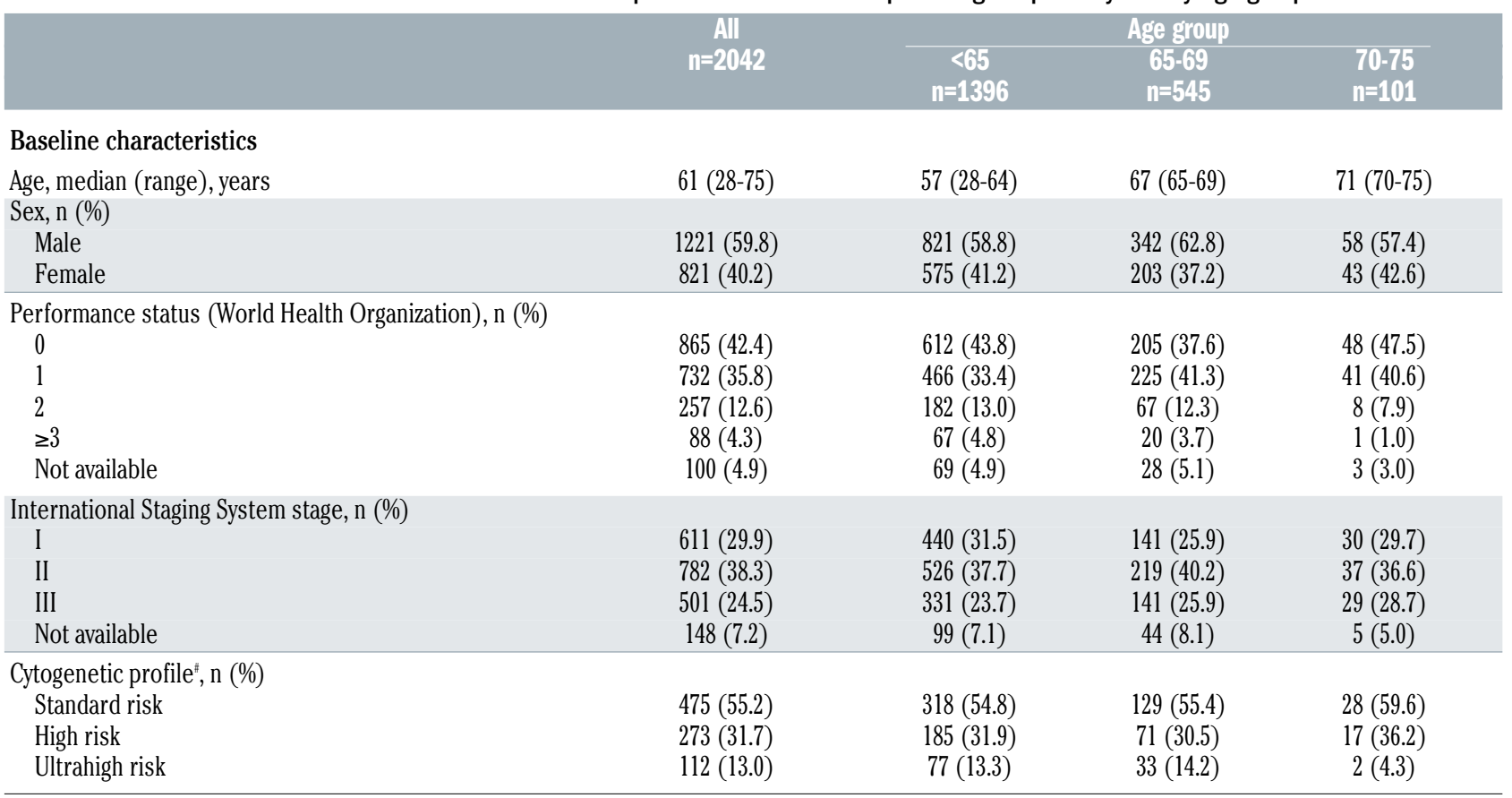

\section{Treatment within TE pathway}

Induction randomisation treatment arm, $\mathrm{n}(\%)$

$\begin{array}{llrrr}\text { CTD } & 1021(50.0) & 701(50.2) & 273(50.1) & 47(46.5)\end{array}$

atients' response at end of Induction (+intensification

$1021(50.0)$

695(49.8) $\quad 272(49.9)$

54 (53.5)

when received), n (\%)

Complete response

Very good partial response

$149(7.3)$

Partial response

Minimal response

$1125(55.1)$

$513(25.1)$

No change

$60(2.9)$

Progressive disease

$16(0.8)$

$44(2.2)$

Unable to assess

$23(1.1)$

No induction treatment or non- protocol treatment

$17(0.8)$

Death within 60 days of initial randomisation or within 60 of

$36(1.8)$

intensification

Not available

$59(2.9)$

$102(7.3)$

$769(55.1)$

$355(25.4)$

$37(2.7)$

$12(0.9)$

$30(2.2)$

$13(0.9)$

$12(0.9)$

$22(1.6)$

$72(49.9)$

Patients who underwent stem cell harvest, n (\%)

No

Unknown

$423(69.7)$

565 (27.7)

$54(2.6)$

$44(3.2)$

$37(6.8)$

$10(9.9)$

$297(54.5)$

$59(58.4)$

$137(25.1)$

$21(20.8)$

$20(3.7)$

$4(0.7)$

$10(1.8)$

$3(3.0)$

$0(0.0)$

$8(1.5)$

$4(4.0)$

$2(2.0)$

$5(0.9)$

$0(0.0)$

$13(2.4)$

$1(1.0)$

Reasons stem cell harvest not performed, $\mathrm{n}(\%)$

Patient decision

Patient not fit/clinicians decision

$129(22.8)$

$200(35.4)$

Disease progression

Death

70 (12.4)

58 (10.3)

$8(1.4)$

Allogeneic transplant

$100(17.7)$

$1026(73.5)$

14(2.6)

$1(1.0)$

Other

$4.4(0.0-90.2)$

332 (23.8)

38 (2.7)

339 (62.2)

$192(35.2)$

58 (57.4)

(2.7)

$14(2.6)$

$41(40.6)$

$2(2.0)$

Median number of $\mathrm{CD}^{2} 4^{+}$cells harvested $\times 10^{6} / \mathrm{kg}$ (range)

Patients achieving $\mathrm{CD} 34^{+}$stem cell harvest thresholds, n (\%)*:

$\geq 2 \times 10^{6} / \mathrm{kg}$
$\geq 4 \times 10^{6} / \mathrm{kg}$

$1277(93.6)$

$791(58.0)$

70 (21.1)

$102(30.7)$

$48(25.0)$

$11(26.8)$

$52(15.7)$

$33(9.9)$

8 (2.4)

$67(20.2)$

$81(42.2)$

17 (41.5)

$13(6.8)$

5 (12.2)

$23(12.0)$

$0(0.0)$

$2(4.9)$

$27(14.1)$

$0(0.0)$

$4.6(0.0-88.8)$

6 (14.6)

Patients who received melphalan (\% of all patients in age group)

$1370(67.1)$

$41(95.0)$

$3.8(0.0-90.2)$

$3.1(0.0-7.7)$

Dose of melphalan administered (\% of all patients who

received melphalan), $\mathrm{n}(\%)$

$70 \mathrm{mg} / \mathrm{m}^{2}$
$100 \mathrm{mg} / \mathrm{m}^{2}$
$140 \mathrm{mg} / \mathrm{m}^{2}$
$200 \mathrm{mg} / \mathrm{m}^{2}$
Unknown/other dose

$1(0.1)$

$14(1.0)$

144 (10.5)

$1161(84.7)$

$50(3.6)$

628 (63.4)

993 (71.1)

$0(0)$

$8(0.8)$

55 (5.5)

895 (90.1)

35 (3.5)
$289(90.0)$

146 (45.6)

47 (88.7)

$322(59.1)$

17 (32.1)

55 (54.5)

$1(1.8)$

$1(1.8)$

$5(1.6)$

$64(19.9)$

239 (74.2)

25 (45.5)

27 (49.1)

1 (1.8) 
Patients who received a melphalan dose and stem cell return (\% of all patients in age group), n (\%)

$1366(66.9)$

Patients response after-ASCT (\% of all patients who received a melphalan dose and stem cell return), $\mathrm{n}(\%)$

Complete response

Very good partial response

Partial response

Minimal response

Progressive disease

Unable to assess

Death up to and including 100 days after ASCT

Not available
$297(21.7)$

$798(58.4)$

$201(14.7)$

$4(0.3)$

$26(1.9)$

$10(0.7)$

$9(0.7)$

$21(1.5)$
$990(70.9)$

$225(22.7)$

$579(58.5)$

135 (13.6)

$4(0.4)$

$19(1.9)$

$8(0.8)$

$5(0.5)$

15 (1.5)
321 (58.9)

55 (54.5)

$\begin{array}{cc}62(19.3) & 10(18.2) \\ 186(57.9) & 33(60.0) \\ 60(18.7) & 6(10.9) \\ 0(0.0) & 0(0.0) \\ 4(1.2) & 3(5.5) \\ 0(0.0) & 2(3.6) \\ 3(0.9) & 1(1.8) \\ 6(1.9) & 0(0.0)\end{array}$

\# data available for 860 of 2,042 (42.1\%) patients (580 of 1,396 [41.5\%] patients aged <65 years, 233 of 545 [42.8\%] patients $65-69$ years and 47 of 101 [46.5\%] patients $70-75$ years) The percentages given are of those with available data. * data available for 1,364 of the 1,423 patients who underwent stem cell harvest (991 of 1,026 patients aged $<65$ years, 320 of 339 patients aged 65-69 years, 53 of 55 patients aged 70-75 years). The percentages given are of those with data available. TE: transplant-eligible; CTD: cyclophosphamide, thalidomide and dexamethasone; CRD: cyclophosphamide, lenalidomide and dexamethasone; ASCT: autologous stem cell transplantation..

ASCT were compared. Patients of different ages achieved a similar depth of response at 100 days after ASCT (Table 1) with an improvement in response compared to the end of induction seen in 863 of $1,366(63.2 \%)$ patients overall $(62.7 \%$ of those aged $<65,64.5 \%$ of those aged $65-69$ and $63.6 \%$ of those aged over 70 ).

The median PFS was longest for patients aged under 65 and fell with increasing age (Figure 1). The median PFS was 50.8 months (95\% CI: 46.3-54.9) for those aged $<65$ years, 40.0 months (95\% CI: 36.3-46.0) for those aged 65-69 and 34.4 months (95\% CI: 27.5-46.4) for those aged $70-75$ years. The PFS for patients aged 65-69 years was shorter than that of patients aged under $65(\mathrm{HR}=1.26, P=0.003)$. Patients aged 70 years or over had a shorter PFS than the $<65$-year age group ( $\mathrm{HR}=1.57, P=0.009$ ), but not significantly shorter than that of the 65-69 age group ( $\mathrm{HR}=1.24, P=0.229)$.

The median OS was 95.5 months (95\% CI: 89.8-not reached) for those aged $<65$ years, 91.9 months $(95 \% \mathrm{CI}$ : 82.3-not reached) for those aged 65-69 and 76.0 months (95\% CI: 58.7-not reached) for those aged 70-75 years. There was no significant difference in the OS between any of the age groups (65-69 vs. $<65$ : $\mathrm{HR}=1.09, P=0.484 ;>70$ vs. <65: $\mathrm{HR}=1.59, P=0.051 ; 70-75$ vs. $65-69, \mathrm{HR}=1.47$, $P=0.127)$. The 5 -year OS was $75.5 \%$ (95\% CI: $72.6 \%-$ $78.3 \%$ ) for those aged <65 years, $72.7 \%$ (95\% CI: $67.3 \%-$ $78.1 \%$ ) for those aged $65-69$ and $65.0 \%$ (95\% CI: $49.5 \%-$ $80.5 \%$ ) for those aged $70-75$ with some evidence of dissociation of the survival curve for the 70-75 age group after 3 years. There was no strong evidence of a difference in OS outcome when accounting for population-level mortality risk (excess mortality hazard-rate ratio [EHR] for OS 65-69 vs. $<65$ : $\mathrm{EHR}=0.95, P=0.736 ; 70-75$ vs. $<65: \mathrm{EHR}=1.33$, $P=0.368$ ) (Online Supplementary Figures $S 2$ and $S 3$, Online Supplementary Table S2). There were also no differences in the percentages of patients who were reported as having commenced second-line therapy at the time of data cut-off (<65: 43.7\%, 65-69: 49.5\% and 70-75: 41.8\%). However, there were notable differences in the proportions of patients whose second-line therapy included a second ASCT $(23.8 \%, 9.4 \%$ and $8.7 \%$, respectively).

Survival outcomes were explored stratified by melphalan dose (Online Supplementary Figure S4). Taking the whole TE population, the $140 \mathrm{mg} / \mathrm{m}^{2}$ dose of melphalan appeared to be associated with a shorter PFS compared to the 200 $\mathrm{mg} / \mathrm{m}^{2}$ dose $(\mathrm{HR}=1.31, P=0.012)$, with no difference in $\mathrm{OS}$ $(\mathrm{HR}=1.29, P=0.086)$ (Online Supplementary Figure $S 4 A)$. However, this result was confounded by age as there were more patients who received $140 \mathrm{mg} / \mathrm{m}^{2}$ in the older age group which was associated with inferior PFS (Figure 1). When examined within each of the age groups there was no difference between outcome for patients who received $140 \mathrm{mg} / \mathrm{m}^{2}$ in comparison to those who received 200 $\mathrm{mg} / \mathrm{m}^{2}$ : age <65: PFS $\mathrm{HR}=1.20, P=0.289$; OS $\mathrm{HR}=1.35$, $P=0.189$ (Online Supplementary Figure S4B); age 65-69: PFS $\mathrm{HR}=1.18, \quad P=0.344 ; \quad \mathrm{OS} \quad \mathrm{HR}=0.97, \quad P=0.905 \quad$ (Online Supplementary Figure S4C); and age $>70$ : PFS $\mathrm{HR}=1.35$, $P=0.442 ; O S H R=1.42, P=0.522$ (Online Supplementary Figure $S 4 D)$.

Transplant-related morbidity and mortality were examined by comparing serious adverse events reported within 100 days of ASCT and deaths occurring within 100 days or 365 days in the different age groups. There were 230 serious adverse events reported within 100 days of ASCT: 172 events in 149 patients in the age group <65 years old (15.1\% of patients), 54 events in 47 patients in the $65-69$ age group $(14.6 \%)$ and four events in four patients in the 70-75 age group $(7.3 \%)$. Nine patients died within 100 days of ASCT: five aged $<65(0.5 \%)$, three aged $65-69(0.9 \%)$ and one aged $>70(1.8 \%)$. Forty-eight patients died within 365 days of ASCT: 34 who were $<65$ years old $(3.4 \%), 11$ who were $65-69(3.4 \%)$ and three who were $70-75$ years old $(5.5 \%)$. These data suggest that there is not a significant increase in mortality or morbidity after ASCT in older patients.

\section{Outcomes in an age-matched group of older patients comparing transplantation to no transplantation}

Analysis of the patients in the TE pathway undergoing transplantation by age does not address the question of whether, at older ages, ASCT continues to be associated with better outcomes than those of patients of an equivalent age not undergoing ASCT. In order to answer this question, an age-matched group of patients was identified as described above and shown in Figure 2A. At baseline, patients in both the TNE and TE-noASCT groups had higher performance status and ISS stage than patients in the TEASCT group (Table 2). Response at the end of induction therapy was deeper in the patients in the TE-ASCT group than in the other two groups.

Older patients undergoing ASCT (TE-ASCT) had a longer median PFS than that of age-matched patients not undergoing ASCT, whether TE-noASCT or TNE (Figure 2B). The median PFS for the TE-ASCT group was 39.4 months compared to 9.7 months for the TE-noASCT group and 16.5 months for the TNE group. Comparing those patients who underwent ASCT (TE-ASCT) to those who did not (TE- 
A

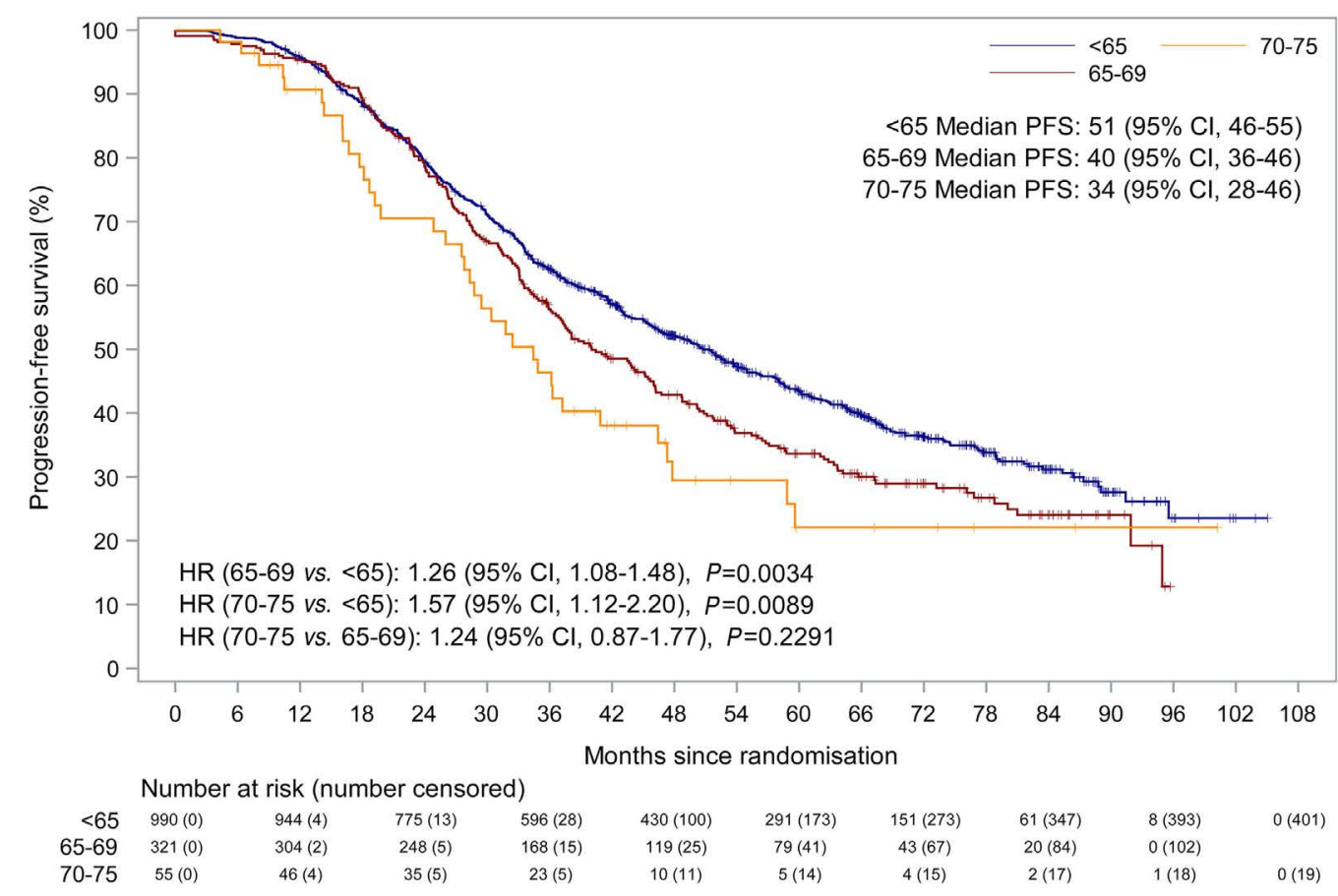

B

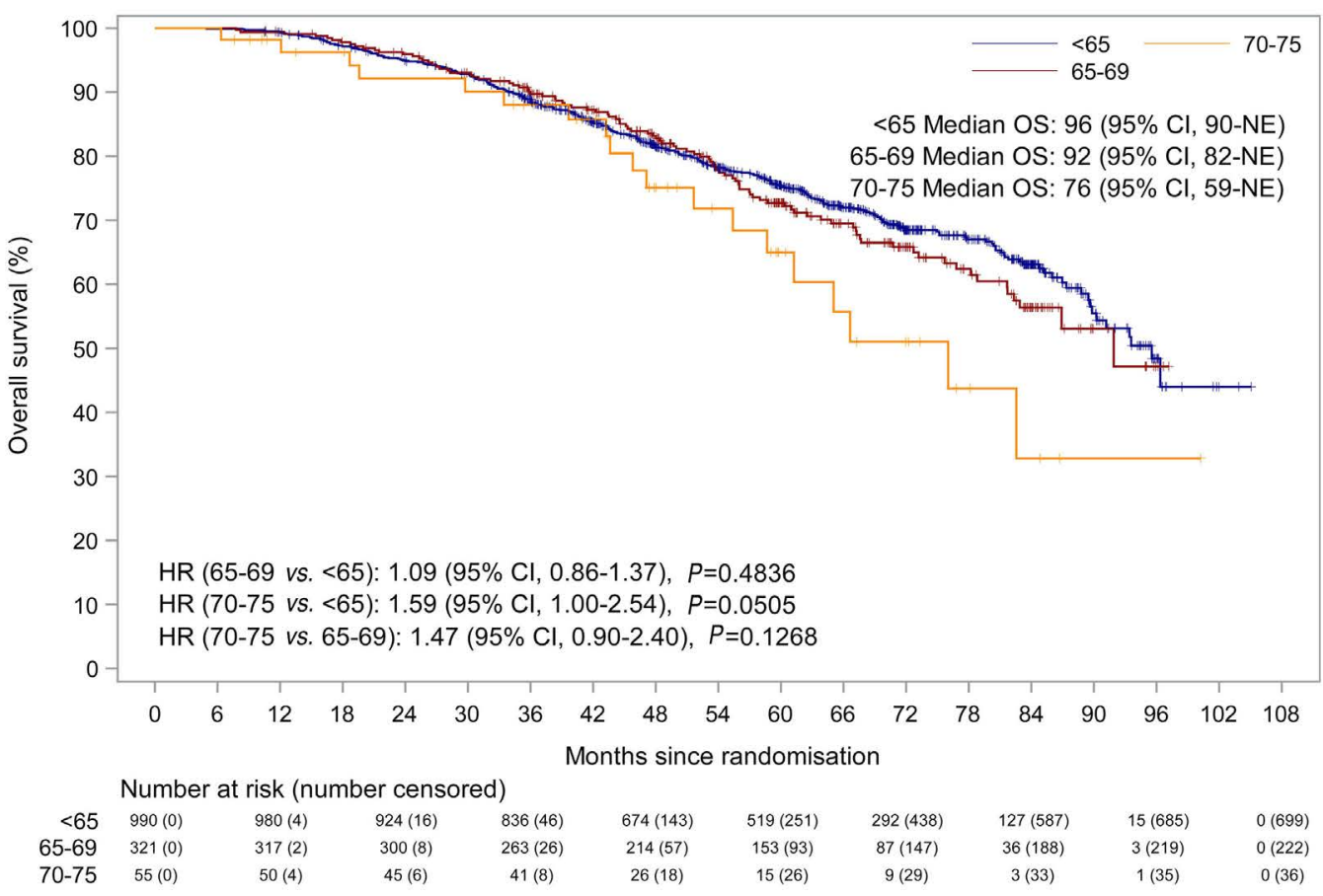

Figure 1. Outcomes of patients of different ages undergoing autologous stem cell transplantation. (A, B) Progression-free survival (A) and overall survival (B) of patients in the groups aged <65 years (blue), 65-69 years (red) and 70-75 years (yellow). PFS: progression-free survival; OS: overall survival; HR: hazard ratio; 95\% Cl: $95 \%$ confidence interval.

noASCT or TNE), ASCT was associated with a significant improvement in PFS (ASCT vs. noASCT: HR=0.41, $P<0.0001)$ (Figure 2D). The same benefit was seen in terms of OS: TE-ASCT median 84.1 months, TE-noASCT 50.9 months, TNE 60.2 months (Figure 2C) (ASCT vs. noASCT: $H R=0.51, P<0.0001$ ) (Figure $2 E$ ). The benefit of ASCT was independent of the subsequent use of mainte- nance therapy, with longer PFS and OS seen in the TEASCT group than in the TNE group whether patients were randomized to observation or maintenance therapy (Online Supplementary Figure S5).

Where possible a frailty-surrogate score was derived for patients in each of the age-matched groups using the UK Myeloma Research Alliance Risk Profile (MRP) $)^{17}$ (Table 2). 
A

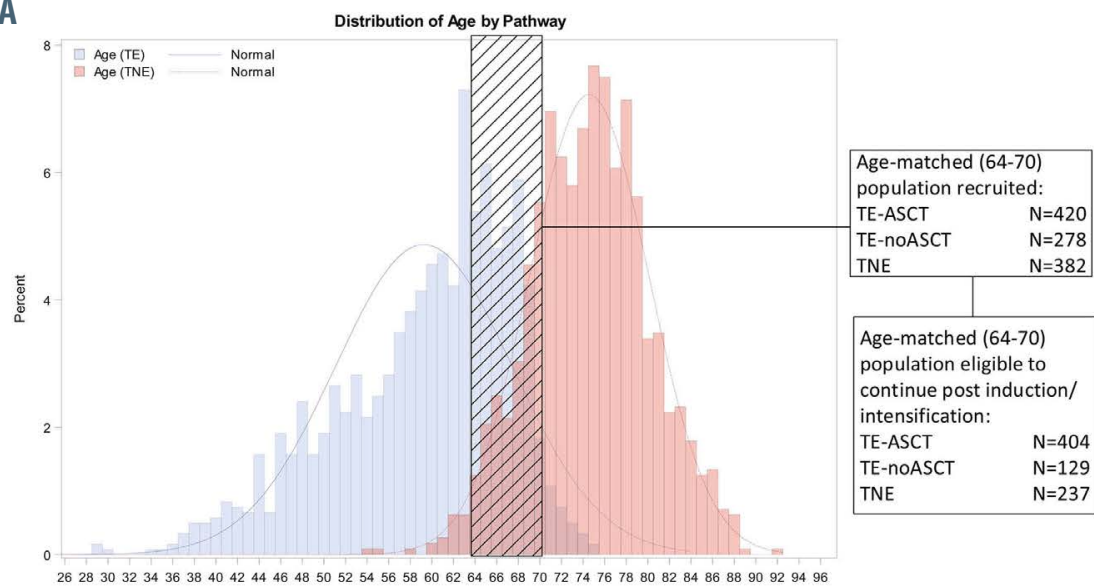

Figure 2. Outcomes of patients in agematched groups. (A) Histogram showing the age distribution of patients in the transplanteligible and transplant-ineligible pathways with the patients included in the age-matched groups highlighted. (B,C) Progression-free survival (B) and overall survival (C) of the agematched population (TE-ASCT [blue], patients in the TE pathway who underwent autologous stem cell transplant; TE-noASCT [red] patients in the TE pathway who did not undergo ASCT; TNE [yellow], patients in the transplant-ineligible pathway. (D,E) Progressionfree survival (D) and overall survival (E) comparing those patients who underwent ASCT with those who did not (ASCT [blue], no ASCT. TE: transplant eligible; TNE: transplant ineligible; HR: hazard ratio; $95 \% \mathrm{Cl}$ : 95\% confidence interval.
B

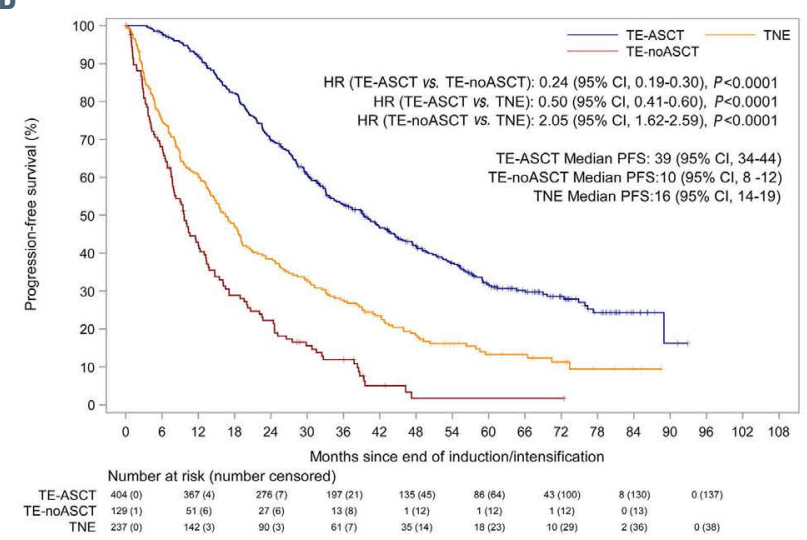

D

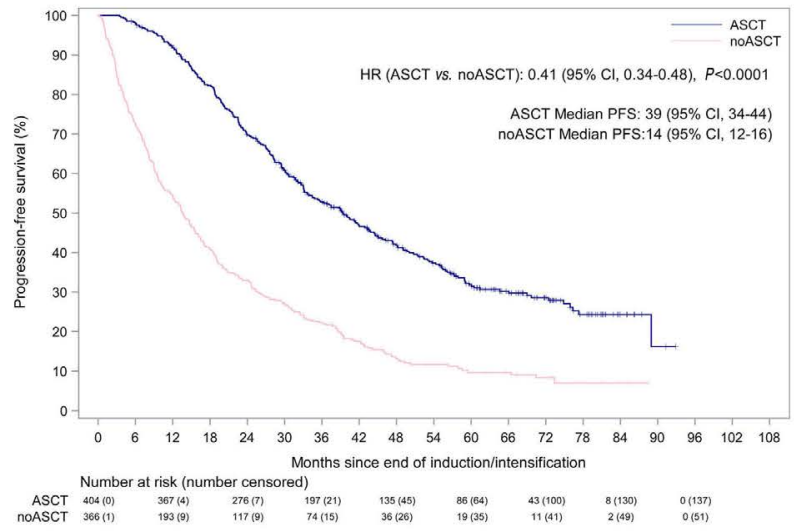

The TE-ASCT group had most patients with a low-risk MRP score and least with a high-risk score compared to the other groups.

The apparent differences in baseline variables and endof-induction responses may have confounded the comparisons between groups. To compensate for this propensity score, IPTW was used to adjust the estimate of the treatment effect of ASCT compared to noASCT in the age-matched group of patients. As expected, the adjustment had the effect of reducing the median PFS and OS for patients in the TE-ASCT group and increasing the median PFS and OS for the patients in the other two groups as compared to the values emerging from the unweighted intention-to-treat analysis. After adjustment,
C

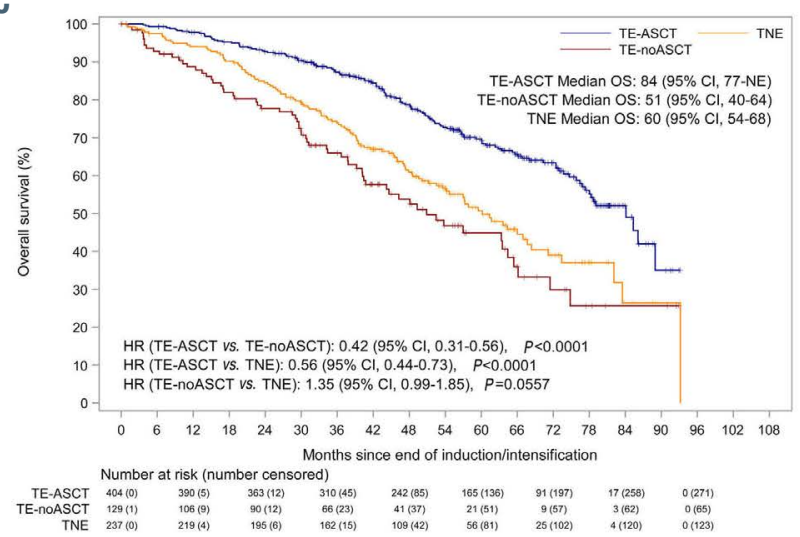

$E$

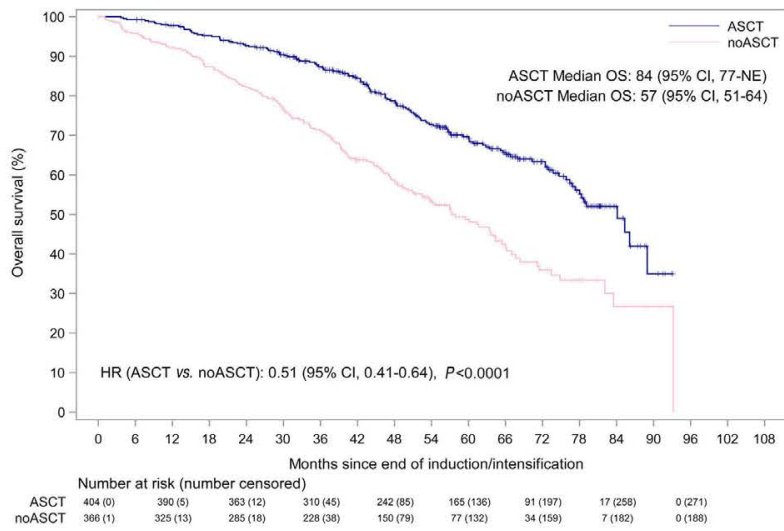

the median PFS for the TE-ASCT group was 35.8 months compared to 10.4 months for the TE-noASCT group and 16.9 months for the TNE group (Figure 3A). Comparing the impact of ASCT versus no ASCT, the hazard ratio remained statistically significant $(\mathrm{HR}=0.44, P<0.001)$. The same benefit was seen in terms of OS, with the median OS for the TE-ASCT group being 79.8 months compared to 57.3 months for the TE-noASCT group and 59.5 months for the TNE group (Figure 3B) (ASCT vs. noASCT: $H R=0.53, P<0.001)$. This analysis suggests that even when the measured baseline covariates were appropriately weighted, there remained a significant treatment benefit of ASCT as compared to no ASCT.

Morbidity and mortality were examined by comparing 
Table 2. Baseline characteristics of patients in the age-matched groups.

\begin{tabular}{|c|c|c|c|c|}
\hline & $\begin{array}{c}\text { All } \\
n=770\end{array}$ & $\begin{array}{l}\text { TE-ASCT } \\
n=404\end{array}$ & $\begin{array}{c}\text { ge-matched groups } \\
\text { TE-noASCT } \\
n=129\end{array}$ & $\begin{array}{c}\text { TNE } \\
n=237\end{array}$ \\
\hline Age, years; median (range) & $67.0(64.0-70.0)$ & $66.0(64.0-70.0)$ & $67.0(64.0-70.0)$ & $68.0(64.0-70.0)$ \\
\hline $\begin{array}{l}\text { Sex, } n(\%) \\
\text { Male } \\
\text { Female }\end{array}$ & $\begin{array}{l}471(61.2) \\
299(38.8)\end{array}$ & $\begin{array}{l}264(65.3) \\
140(34.7)\end{array}$ & $\begin{array}{l}73(56.6) \\
56(43.4)\end{array}$ & $\begin{array}{l}134(56.5) \\
103(43.5)\end{array}$ \\
\hline $\begin{array}{l}\text { Performance status, (WHO), n (\%) } \\
\quad 0 \\
1 \\
2 \\
\geq 3 \\
\quad \text { Not available }\end{array}$ & $\begin{array}{c}269(34.9) \\
321(41.7) \\
109(14.2) \\
34(4.5) \\
37(4.8)\end{array}$ & $\begin{array}{l}160(39.6) \\
173(42.8) \\
41(10.1) \\
7(1.7) \\
23(5.7)\end{array}$ & $\begin{array}{c}44(34.1) \\
51(39.5) \\
20(15.5) \\
10(7.8) \\
4(3.1)\end{array}$ & $\begin{array}{l}65(27.4) \\
97(40.9) \\
48(20.3) \\
17(7.2) \\
10(4.2)\end{array}$ \\
\hline $\begin{array}{l}\text { International Staging System, n (\%) } \\
\text { I } \\
\text { II } \\
\text { III } \\
\text { Not available }\end{array}$ & $\begin{array}{c}199(25.8) \\
318(41.3) \\
195(25.3) \\
58(7.5)\end{array}$ & $\begin{array}{l}125(30.9) \\
164(40.6) \\
79(19.6) \\
36(8.9)\end{array}$ & $\begin{array}{c}29(22.5) \\
52(40.3) \\
43(33.3) \\
5(3.9)\end{array}$ & $\begin{array}{l}45(19.0) \\
102(43.0) \\
73(30.8) \\
17(7.2)\end{array}$ \\
\hline $\begin{array}{l}\text { Cytogenetic profile, n (\%) } \\
\text { Standard risk } \\
\text { High risk } \\
\text { Ultrahigh risk }\end{array}$ & $\begin{array}{l}184(55.9) \\
105(31.9) \\
40(12.2)\end{array}$ & $\begin{array}{l}86(51.8) \\
55(33.1) \\
25(15.1)\end{array}$ & $\begin{array}{l}37(57.8) \\
19(29.7) \\
8(12.5)\end{array}$ & $\begin{array}{c}61(61.6) \\
31(31.3) \\
7(7.1)\end{array}$ \\
\hline $\begin{array}{l}\text { Induction Randomisation Treatment, n (\%) } \\
\text { CTD/CTDa } \\
\text { CRD/CRDa }\end{array}$ & $\begin{array}{l}345(44.8) \\
425(55.2)\end{array}$ & $\begin{array}{l}194(48.0) \\
210(52.0)\end{array}$ & $\begin{array}{l}62(48.1) \\
67(51.9)\end{array}$ & $\begin{array}{c}89(37.6) \\
148(62.4)\end{array}$ \\
\hline $\begin{array}{l}\text { MRP possible to define, n (\%) } \\
\text { Yes } \\
\text { No }\end{array}$ & $\begin{array}{l}646(83.9) \\
124(16.1)\end{array}$ & $\begin{array}{l}303(75.0) \\
101(25.0)\end{array}$ & $\begin{array}{l}106(82.2) \\
23(17.8)\end{array}$ & $\begin{array}{c}237(100) \\
0(0)\end{array}$ \\
\hline $\begin{array}{l}\text { MRP risk (\% based on those patients } \\
\text { with all MRP data available), n (\%) } \\
\text { Low } \\
\text { Intermediate } \\
\text { High }\end{array}$ & $\begin{array}{c}430(66.6) \\
152(23.5) \\
64(9.9)\end{array}$ & $\begin{array}{c}243(80.2) \\
47(15.5) \\
13(4.3)\end{array}$ & $\begin{array}{l}60(56.6) \\
26(24.5) \\
20(18.9)\end{array}$ & $\begin{array}{l}127(53.6) \\
79(33.3) \\
31(13.1)\end{array}$ \\
\hline $\begin{array}{l}\text { Patients' response after Induction } \\
\text { (+intensification where received), n (\%) } \\
\text { Complete response } \\
\text { Very good partial response } \\
\text { Partial response } \\
\text { Minimal response }\end{array}$ & $\begin{array}{c}94(12.2) \\
554(72.0) \\
114(14.8) \\
8(1.0)\end{array}$ & $\begin{array}{c}51(12.6) \\
286(70.8) \\
65(16.1) \\
2(0.5)\end{array}$ & $\begin{array}{c}10(7.8) \\
97(75.2) \\
19(14.7) \\
3(2.3)\end{array}$ & $\begin{array}{c}33(13.9) \\
171(72.2) \\
30(12.7) \\
3(1.3)\end{array}$ \\
\hline
\end{tabular}

serious adverse events and deaths reported within 100 days of the end of induction ( \pm intensification). Two hundred and three serious adverse events were reported within 100 days: 132 events in 105 patients in the TEASCT group (26.0\% of patients), 70 events in 49 patients in the TE-noASCT group (38.0\%) and 65 events in 53 patients in the TNE group $(22.4 \%)$. Five patients died within 100 days: none in the TE-ASCT group, two in the TE-noASCT group $(2.7 \%)$ and three in the TNE group (2.18\%). Thirty-seven patients died within 365 days: nine in the TE-ASCT group $(2.2 \%), 14$ in the TE-noASCT group $(10.9 \%)$ and 14 in the TNE group (5.9\%).

\section{Discussion}

These results demonstrate that ASCT is safe and effective for selected, fit, myeloma patients up to the age of 75 . In an age-matched population treated with similar induction therapy there was a significant benefit for PFS and OS associated with the use of ASCT compared to no ASCT.

The study showed that even in a group of patients initially felt to be eligible for transplantation by their treating clinician, there was a clear fall in the proportion of patients undergoing stem cell harvest and ASCT with increasing age. This likely reflects clinicians' enthusiasm for giving patients the option of having an ASCT, by enrolling the patients in the TE pathway, but a subsequent realization that the patients were not fit enough. Commencing intensive induction therapy and using this as a therapeutic trial of fitness before making the final decision regarding ASCT may represent a valid approach to therapy especially in the intermediate age group of those aged 65-75 years.

Although the median $\mathrm{CD}_{3} 4^{+}$harvest cell count was lower in older patients it is not certain whether this reflects a true difference in mobilization. The percentage of patients from whom enough stem cells were collected for one ASCT $\left(2 \times 10^{6} \mathrm{CD}_{3} 4^{+}\right.$cells $\left./ \mathrm{kg}\right)$ was very high across 
A

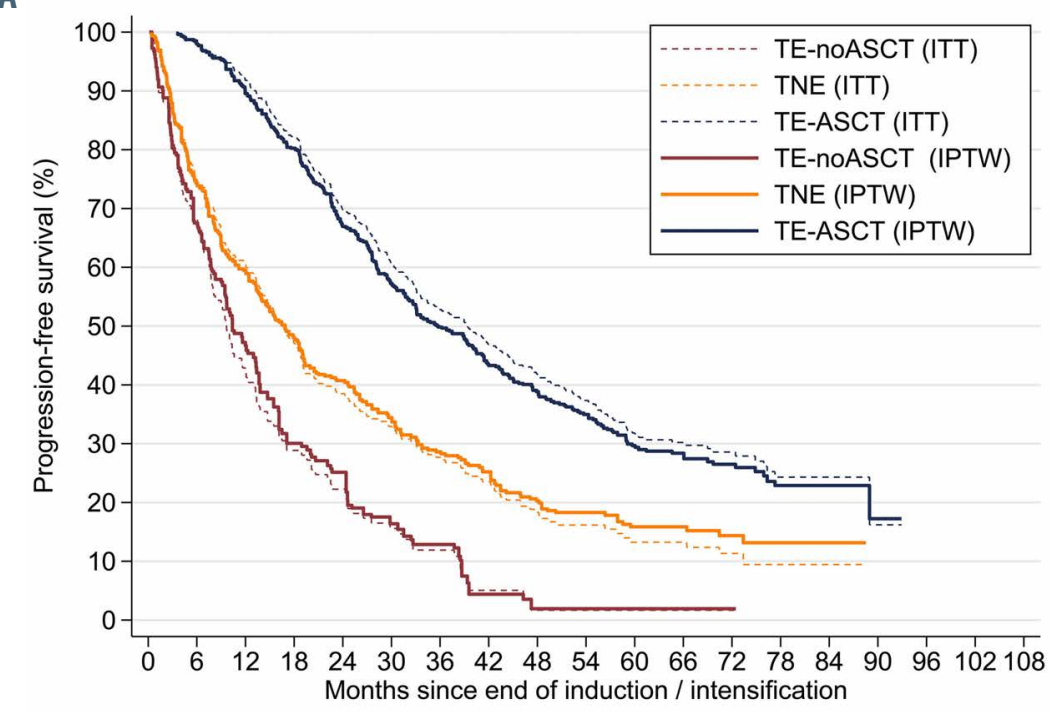

B

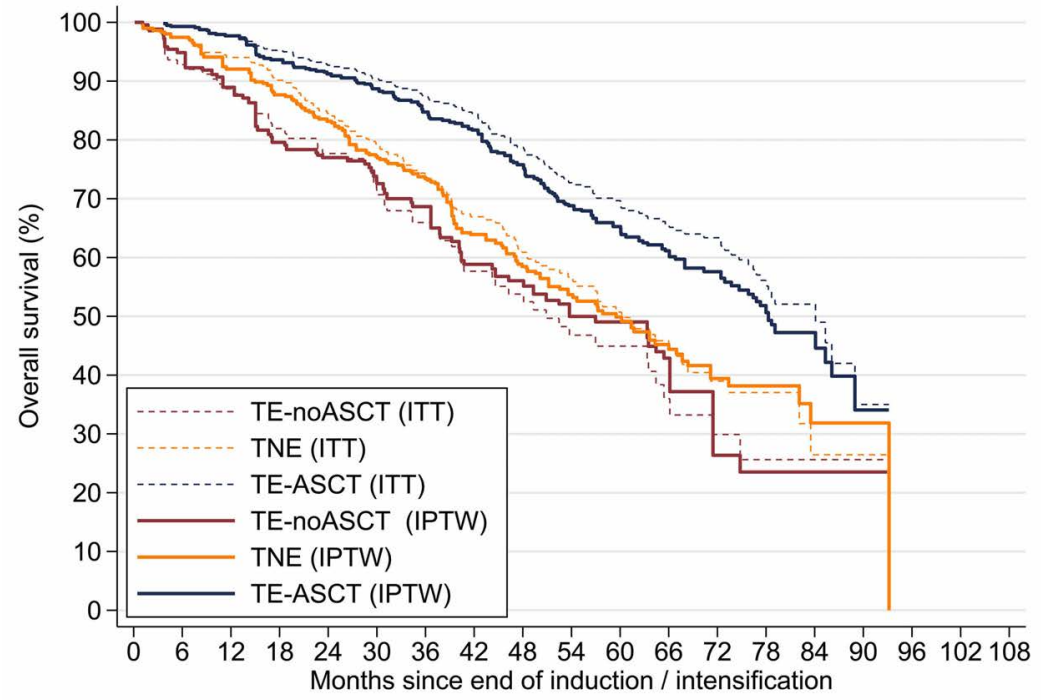

Figure 3. Outcomes of patients in age-matched groups including inverse probability of treatment weighting. (A) Progression-free survival. (B) Overall survival. TE-ASCT (blue): patients in the transplanteligible pathway who underwent autologous stem cell transplantation; TE-noASCT (red): patients in the transplant-eligible pathway who did not undergo autologous stem cell transplantation; TNE (yellow): patients in the transplant-ineligible pathway; ITT: intention to treat; IPTW: inverse probability of treatment weighting adjustment. the age groups. It is unknown what the local investigators set as the target harvest for each patient and it may be that the target for older patients was to collect enough stem cells for one transplant rather than to also save some for a possible subsequent transplant given that by the time of relapse the older patients would have achieved an even more advanced age.

The PFS of patients in the TE pathway aged 65-69 and those aged 70-75 was shorter than that of patients aged under 65 years. This would be expected as outcomes are known to diminish with increasing age with all myeloma therapies. There was no significant difference in OS, although the survival curves appeared to dissociate for the 70-75 age group after 3 years. To further investigate this we performed OS analysis corrected for population-level mortality risk and found no evidence of a difference in survival. ASCT delivery to selected older patients was safe; there was no difference in survival at 3 months or 1 year after ASCT between age groups. Indeed, fewer serious adverse events occurred within 100 days of ASCT among those in the oldest age group. This may be due to the small size of this age group or due to more stringent selection for fitness in these older patients.

There was no significant difference in PFS or OS in this study when comparing patients of a similar age who received $140 \mathrm{mg} / \mathrm{m}^{2}$ melphalan (due to renal impairment or clinician choice) or $200 \mathrm{mg} / \mathrm{m}^{2}$ melphalan as conditioning for ASCT. This reinforces the approach of using a dose reduction of melphalan conditioning only in these selected subsets of patients, with no apparent detriment to outcomes. One previous study suggested that there was increased toxicity with the higher dose in those aged over 70 years ${ }^{24}$ but a much larger and more recent study of the EBMT Registry database did not reveal any significant difference in survival outcomes between the groups receiving different doses in the overall population, but a benefit from the use of $200 \mathrm{mg} / \mathrm{m}^{2}$ in those with a suboptimal response. ${ }^{25}$ As in our study, far fewer patients received the $140 \mathrm{mg} / \mathrm{m}^{2}$ dose than the $200 \mathrm{mg} / \mathrm{m}^{2}$ dose in the EBMT analysis, suggesting that the lower dose was only used in 
very selected older patients or in those with renal failure. In the Myeloma XI trial the induction therapy for TE and TNE patients was the same triplet combination. This provided the opportunity to compare outcomes for those patients undergoing transplantation with patients of the same age who did not undergo transplantation but who had received the same induction therapy. We performed this analysis from the end of induction ( \pm intensification if given) and only included patients who would have been eligible to continue in the study to avoid survivor and immortal time biases. This comparison showed a marked improvement in PFS and OS associated with ASCT. It is important to note that this comparison between ASCT and no ASCT was not randomized and therefore remains inherently subject to bias. Patients in the TE pathway of the trial were selected on the basis of clinician's judgement and patient's preference. We found that the older patients included in the TE pathway had a lower performance status than younger patients, whereas performance status usually increases with age. This suggests active selection of only the fittest older patients for entry into the TE pathway and consideration of ASCT. Consistent with this, in the age-matched population the performance status and ISS stage were higher in both the TE-noASCT and TNE groups than in the TE-ASCT patients. Unfortunately, data to calculate the International Myeloma Working Group frailty score, ${ }^{26}$ the Revised Myeloma Comorbidity Index ${ }^{27}$ or the Hematopoietic Cell Transplant Comorbidity Index ${ }^{28}$ were not collected within the study. We applied the UKMRP, an outcome score previously validated only in the TNE population, across the groups and found more patients in the TNE and TE-noASCT groups to have higher-risk MRP than in the TE-ASCT group. Additionally, the TE-ASCT group had achieved deeper responses at the end of induction. This may have affected their outcomes irrespective of transplantation. To address this, a matched analysis using IPTW was performed with factors including those associated with frailty and response to induction that were different between the age-matched groups. This analysis confirmed the markedly improved PFS and OS for the ASCT-TE group, suggesting that this finding was not confounded by fitness or prior response. It should be noted that propensity scores only balance measured covariates as confounders, and balance in measured covariates does not necessarily indicate balance in unmeasured confounders. If unmeasured covariates are confounders, they can bias treatment effect estimates. These results should therefore be interpreted with caution.

Previous studies in younger, fitter patients under the age of 65 have demonstrated the efficacy of transplantation in the era of modern therapy including studies combining proteasome inhibitors and immunomodulatory agents for all patients as induction therapy. Such approaches may now be considered better than the largely immunomodulatory agent-based induction delivered in Myeloma XI. In the IFM/DFCI 2009 study patients received bortezomib, lenalidomide and dexamethasone (VRD) induction before randomization between ASCT and consolidation in the form of additional cycles of VRD, with ASCT deferred to first relapse. ${ }^{29}$ The trial demonstrated an association between improved PFS and early ASCT while follow-up for $\mathrm{OS}$ is ongoing. The EMN02 trial conducted a similar comparison but in the context of induction with cyclophosphamide, bortezomib and dexamethasone and compared the use of bortezomib, melphalan and pred- nisone (VMP) consolidation to ASCT, ${ }^{6}$ also demonstrating a PFS advantage for first-line ASCT. Our data support the findings of these studies and extend them to older patients who were excluded from these trials. Sub-analysis of IFM/DFCI 2009 suggests that there is no benefit of transplantation in patients achieving very deep minimal residual disease-negative responses prior to ASCT. This is of great interest as it could lead to a response-adapted approach to ASCT. Minimal residual disease data were collected for a subset of the patients within the Myeloma XI trial, but there were too few patients in the agematched population to perform this analysis. Within the last 18 months two large phase III studies showed that the addition of daratumamb to standard induction regimens (lenalidomide-dexamethasone and VMP) dramatically improved the PFS and OS of older patients. ${ }^{30-32}$ TNE patients were randomized into these studies, although the reason for transplant ineligibility (age, co-morbidities) was not stated. Randomized clinical trials are therefore still warranted for older patients who are fit for transplant comparing an antibody-containing regimen \pm transplantation.

In summary, these findings support the use of ASCT for selected, fit older myeloma patients up to the age of 75 . With effective clinician selection, older patients undergoing ASCT can experience long PFS and OS, comparable to those of younger patients, and without any significant increase in morbidity or mortality.

\section{Disclosures}

$C P$ has provided consultancy services for and received travel support from Amgen and Takeda Oncology; honoraria and travel support from Janssen; and consultancy fees, honoraria, and travel support from Celgene Corporation. DAC has received research funding from Celgene Corporation, Amgen, and Merck Sharp and Dohme. TM has received research funding from Celgene Corporation, Amgen, and Merck Sharp and Dohme. JRJ has received honoraria and research funding from Celgene Corporation. MWJ has acted as a consultant for and received honoraria, travel support, and research funding from Janssen; has acted as a consultant for and received honoraria and travel support from Takeda and Amgen; has acted as a consultant for and received honoraria and research funding from Celgene Corporation; and has acted as a consultant for and received honoraria from Novartis. GC has provided consultancy and speakers bureau services for and received honoraria and research funding from Takeda, Celgene Corporation, Janssen and Amgen; has acted as a consultant for and received honoraria from Glycomimetics and Bristo-Myers Squibb; and has provided consultancy and speakers bureau services for and received honoraria from Sanofi. KDB has received honoraria from Celgene, Janssen, and Amgen; acted in a consulting or advisory role for Celgene, Janssen, Takeda, and Novartis; and received travel support from Celgene, Janssen, and Takeda. MTD has equity ownership and membership on the board of directors or advisory committees of Abingdon Health. MFK has acted as a consultant for and received travel support from Bristol-Myers Squibb and Takeda; has acted as a consultant for Chugai; has acted as a consultant for and received honoraria from Janssen and Amgen; and has acted as a consultant for and received honoraria and research funding from Celgene Corporation. RGO has received honoraria and travel support from Takeda; has provided consultancy services for and received travel support from Janssen; and has acted as a consultant for and received honoraria and research funding from Celgene Corporation. WG has provided consultancy services for and 
received research funding from Celgene Corporation; has received research funding from Amgen and Merck Sharp and Dohme; and has received honoraria from Janssen. GJM has received research funding from Janssen; has provided consultancy services for and received honoraria from Bristol-Myers Squibb, Takeda, Roche, Amgen, GSK and Karyopharm; and has acted as a consultant for and received honoraria and research funding from Celgene Corporation. GHJ has provided consultancy and speakers bureau services for and received honoraria from Roche, Amgen, Janssen, and Merck Sharp and Dohme; and has provided consultancy and speakers bureau services for and received honoraria, travel support and research funding from Celgene Corporation and Takeda. FED has received honoraria from Adaptive; has provided consultancy services for and received honoraria and research funding from Celgene Corporation; and has provided consultancy services for and received honoraria from Janssen, Oncopeptide, Roche, Sanofi, and Takeda.

\section{Contributions}

$C P, D A C$ and FED designed this analysis; GJM, GHJ and FED were Chief Investigators of the Myeloma XI trial; $C P$, $K D B, J R J, M W J, G C, M F K, R G O, G J M, G H J$ and FED participated in the recruitment and management of patients; MFK, MTD, RGO and GJM coordinated the central laboratory investigations; CP, DAC, TM and FED analyzed and interpreted the data for this analysis; CP, DAC and TM drafted the manuscript. All authors contributed to critically revising the manuscript and approved the final submitted version.

\section{Acknowledgments}

We thank all the patients at centers throughout the UK whose willingness to participate made this study possible. We are grateful to the UK National Cancer Research Institute Haematological Oncology Clinical Studies Group, UK Myeloma Research Alliance, and to all principal investigators, sub-investigators, and local center staff for their dedication and commitment to recruiting patients to the study. We thank the members of the Myeloma XI Trial Steering Committee and Data Monitoring and Ethics Committee. The support of the Clinical Trials Research Unit at the University of Leeds was essential to the successful running of the study; we thank all their staff who have contributed, past and present. Central laboratory analysis was performed at the Institute of Immunology and Immunotherapy, University of Birmingham; the Institute of Cancer Research, London; and the Haematological Malignancy Diagnostic Service, St James's University Hospital, Leeds. We are very grateful to the laboratory teams for their contribution to the study. We also acknowledge support from the National Institute of Health Biomedical Research Centre at the Royal Marsden Hospital and the Institute of Cancer Research.

\section{Funding}

Primary financial support was from Cancer Research UK (C1298/A10410). Unrestricted educational grants from Celgene Corporation, Amgen, and Merck Sharp and Dohme, and funding from Myeloma UK supported trial coordination and laboratory studies. The authors are solely responsible for the study design, data collection, data analysis and interpretation, writing, and decisions about publication submission; no funder had any role in these aspects of the trial. Trial data were accessible to all authors.

\section{Data-sharing statement}

De-identified participant data will be made available when all primary and secondary endpoints of the trial have been met. Any requests for trial data and supporting material (data dictionary, protocol, and statistical analysis plan) will be reviewed by the trial management group in the first instance. Only requests that have a methodologically sound basis and whose proposed use of the data has been approved by the independent trial steering committee will be considered. Proposals should be directed to the Chief Investigator responsible for trial governance, Prof. Graham Jackson in the first instance; to gain access, data requestors will need to sign a data access agreement.

\section{References}

1. Child JA, Morgan GJ, Davies FE, et al. Highdose chemotherapy with hematopoietic stem-cell rescue for multiple myeloma. $\mathrm{N}$ Engl J Med. 2003;348(19):1875-1883.

2. Attal M, Harousseau JL, Stoppa AM, et al. A prospective, randomized trial of autologous bone marrow transplantation and chemotherapy in multiple myeloma. Intergroupe Francais du Myelome. N Engl J Med. 1996;335(2):91-97.

3. Fermand JP, Katsahian S, Divine $M$, et al. High-dose therapy and autologous blood stem-cell transplantation compared with conventional treatment in myeloma patients aged 55 to 65 years: long-term results of a randomized control trial from the Group Myelome-Autogreffe. J Clin Oncol. 2005;23(36):9227-9233

4. Bladé J, Rosiñol L, Sureda A, et al. High-dose therapy intensification compared with continued standard chemotherapy in multiple myeloma patients responding to the initial chemotherapy: long-term results from a prospective randomized trial from the Spanish cooperative group PETHEMA. Blood. 2005;106(12):3755-3759.

5. Attal M, Lauwers-Cances V, Hulin C, et al. Lenalidomide, bortezomib, and dexamethasone with transplantation for myeloma. $\mathrm{N}$ Engl J Med. 2017;376(14):1311-1320.
6. Cavo M, Gay F, Beksac M, et al. Autologous haematopoietic stem-cell transplantation versus bortezomib-melphalan-prednisone, with or without bortezomib-lenalidomidedexamethasone consolidation therapy, and lenalidomide maintenance for newly diagnosed multiple myeloma (EMN02/HO95): a multicentre, randomised, open-label, phase 3 study. Lancet Haematol. 2020;7(6):e456e468.

7. Auner HW, Szydlo R, Hoek J, et al. Trends in autologous hematopoietic cell transplantation for multiple myeloma in Europe: increased use and improved outcomes in elderly patients in recent years. Bone Marrow Transplant. 2015;50(2):209-215.

8. Munshi PN, Vesole D, Jurczyszyn A, et al. Age no bar: a CIBMTR analysis of elderly patients undergoing autologous hematopoietic cell transplantation for multiple myeloma. Cancer 2020;126(23): 5077-87.

9. Palumbo A, Bringhen S, Petrucci MT, et al. Intermediate-dose melphalan improves survival of myeloma patients aged 50 to 70 : results of a randomized controlled trial. Blood. 2004;104(10):3052-3057.

10. Facon T, Mary JY, Hulin C, et al. Melphalan and prednisone plus thalidomide versus melphalan and prednisone alone or reducedintensity autologous stem cell transplantation in elderly patients with multiple myeloma (IFM 99-06): a randomised trial. Lancet. 2007;370(9594):1209-1218.
11. Muchtar E, Dingli D, Kumar S, et al. Autologous stem cell transplant for multiple myeloma patients 70 years or older. Bone Marrow Transplant. 2016;51(11):1449-1455.

12. Merz M, Neben K, Raab MS, et al Autologous stem cell transplantation for elderly patients with newly diagnosed multiple myeloma in the era of novel agents. Ann Oncol. 2014;25(1):189-195.

13. Biran N, Jacobus S, Vesole DH, et al Outcome with lenalidomide plus dexamethasone followed by early autologous stem cell transplantation in patients with newly diagnosed multiple myeloma on the ECOGACRIN E4A03 randomized clinical trial: long-term follow-up. Blood Cancer J. 2016;6(9):e466.

14. Wildes TM, Finney JD, Fiala M, et al. High dose therapy and autologous stem cell transplant in older adults with multiple myeloma. Bone Marrow Transplant. 2015;50(8): 1075-1082.

15. Jackson GH, Davies FE, Pawlyn C, et al. Lenalidomide maintenance versus observation for patients with newly diagnosed multiple myeloma (Myeloma XI): a multicentre, open-label, randomised, phase 3 trial. Lancet Oncol. 2019;20(1):57-73.

16. Jackson GH, Davies FE, Pawlyn C, et al. Response-adapted intensification with cyclophosphamide, bortezomib, and dexamethasone versus no intensification in patients with newly diagnosed multiple 
myeloma (Myeloma XI): a multicentre, open-label, randomised, phase 3 trial. Lancet Haematol. 2019;6(12):e616-e629.

17. Royston P, Lambert PC. Flexible parametric survival analysis using stata: beyond the Cox model. College Station, Tex. Stata, 2011.

18. https://www.ons.gov.uk/peoplepopulationand community/birthsdeathsandmarriages/lifeexpectancies/datasets/nationallifet ablesunitedkingdomreferencetables. (Last accessed Feb 2017).

19. Cook G, Royle KL, Pawlyn C, et al. A clinical prediction model for outcome and therapy delivery in transplant-ineligible patients with myeloma (UK Myeloma Research Alliance Risk Profile): a development and validation study. Lancet Haematol. 2019;6(3):e154-e166.

20. Boyle EM, Proszek PZ, Kaiser MF, et al. A molecular diagnostic approach able to detect the recurrent genetic prognostic factors typical of presenting myeloma. Genes Chromosomes Cancer. 2015;54(2):91-98.

21. Kaiser MF, Walker BA, Hockley SL, et al. A TC classification-based predictor for multiple myeloma using multiplexed real-time quantitative PCR. Leukemia. 2013;27(8): 1754-1757.

22. Boyd KD, Ross FM, Chiecchio L, et al. A novel prognostic model in myeloma based on co-segregating adverse FISH lesions and the ISS: analysis of patients treated in the MRC Myeloma IX trial. Leukemia. 2012;26(2):349-355.

23. Sonneveld P, Avet-Loiseau H, Lonial S, et al. Treatment of multiple myeloma with highrisk cytogenetics: a consensus of the International Myeloma Working Group. Blood. 2016;127(24):2955-2962.

24. Badros A, Barlogie B, Siegel E, et al. Autologous stem cell transplantation in elderly multiple myeloma patients over the age of 70 years. Br J Haematol. 2001;114(3):600607.

25. Auner HW, Iacobelli S, Sbianchi G, et al. Melphalan $140 \mathrm{mg} / \mathrm{m}(2)$ or $200 \mathrm{mg} / \mathrm{m}(2)$ for autologous transplantation in myeloma: results from the Collaboration to Collect Autologous Transplant Outcomes in Lymphoma and Myeloma (CALM) study. A report by the EBMT Chronic Malignancies Working Party. Haematologica. 2018;103(3): 514-521.

26. Palumbo A, Bringhen S, Mateos MV, et al. Geriatric assessment predicts survival and toxicities in elderly myeloma patients: an International Myeloma Working Group report. Blood. 2015;125(13):2068-2074.

27. Engelhardt M, Domm AS, Dold SM, et al. A concise revised Myeloma Comorbidity Index as a valid prognostic instrument in a large cohort of 801 multiple myeloma patients. Haematologica. 2017;102(5):910921

28. Saad A, Mahindra A, Zhang MJ, et al. Hematopoietic cell transplant comorbidity index is predictive of survival after autologous hematopoietic cell transplantation in multiple myeloma. Biol Blood Marrow Transplant. 2014;20(3):402-408.

29. Attal M, Lauwers-Cances V, Hulin C, et al. Lenalidomide, bortezomib, and dexamethasone with transplantation for myeloma. N Engl J Med. 2017;376(14):1311-1320.

30. Facon $T$, Kumar $S$, Plesner $T$, et al. Daratumumab plus lenalidomide and dexamethasone for untreated myeloma. N Eng J Med. 2019;380(22):2104-2115.

31. Mateos MV, Dimopoulos MA, Cavo M, et al. Daratumumab plus bortezomib, melphalan, and prednisone for untreated myeloma. N Engl J Med. 2018;378(6):518-528.

32. Mateos MV, Cavo M, Blade J, et al. Overall survival with daratumumab, bortezomib, melphalan, and prednisone in newly diagnosed multiple myeloma (ALCYONE): a randomised, open-label, phase 3 trial. Lancet. 2020;395(10218):132-141. 